\title{
A Cluster-based Routing Method for D2D Communication Oriented to Vehicular Networks
}

\author{
Yuhong Li Haoyue Xue Zhihui Gai Xirong Que \\ State Key Laboratory of Networking and Switching Technology \\ Beijing University of Posts and Telecommunications \\ Beijing China
}

\author{
Xiang Su Jukka Riekki \\ Centre for Ubiquitous Computing \\ University of Oulu \\ Oulu, Finland
}

\begin{abstract}
The combination of Device-to-Device (D2D) Communication in 5G Cellular Networks and vehicular networks will not only increase the performance of vehicular networks, but also increase the revenues for network operators and services providers. This paper proposes a 5G D2D routing method oriented to vehicular networks, which can increase the connectivity and scalability of vehicular networks while alleviating the traffic load of $5 \mathrm{G}$ base stations. Vehicular nodes are clustered according to their communication range, the speed and direction of the movements. Data are transferred among vehicles in the same and neighbor clusters in the D2D mode, only certain data packets are needed to be transferred through the 5G base stations. In this way, the traffic load of $5 \mathrm{G}$ based stations can be reduced greatly, whereas the data transmission delay of the vehicular networks can also be guaranteed. Simulation results show that our cluster-based routing mechanism is stable and inexpensive, and is suitable for the vehicular networks.
\end{abstract}

Keywords—vehicular networks; routing; clustering; D2D;5G

\section{INTRODUCTION}

By connecting people with vehicles, vehicles with vehicles, and vehicles with environments, vehicular networks are not only a method for increasing road safety and transport efficiency, and providing infotainment for people in the mobile environment, but also a way for increasing revenues for service providers and car manufacturers. On the one hand, 5G networks have been presented and studied for several years, with the key feature of providing large communication capability for a huge number of devices in a large area. However, applications oriented to $5 \mathrm{G}$ require more research. Vehicular networks, with a large number of nodes moving at high speed, and needing to support various applications with different data rate, can potentially become a major application for $5 \mathrm{G}$, especially the device-to-device (D2D) technique in the context of $5 \mathrm{G}$.

On the other hand, the development and deployment of vehicular networks will also benefit from 5G D2D. The commonly used IEEE 802.11p technique is suitable for short distance, low speed communications among vehicles. Without infrastructure-based networks, only intermittent, short time connectivity can be provided. Large area communication with certain QoS guarantees cannot be realized due to the constraints of roads, obstacles, sparse or dense distribution of vehicles in certain areas and high speed of vehicles, etc. The research of the combination of LTE and VANET [1] has shown the advantages of cellular networks brought to vehicular networks. However, the increasing number of vehicles brings heavy load to the LTE base stations, which may influence the performance of not only the vehicular networks, but also the whole LTE system. D2D is a short distance communication technology assisted by $5 \mathrm{G}$ base stations. By using D2D, the communication area of vehicular networks can be greatly enlarged without deploying Road Side Units (RSUs) which will cost lots of money. With the help of the $5 \mathrm{G}$ base stations, the quality of service of the vehicular networks may also be improved, and the radio resource can also be used efficiently.

Currently, some research has been done on the LTE assisted vehicular networks. However, they are aimed at solving certain problems, such as secure information distribution or the "dead end" problem under sparse vehicular nodes, etc. [2] [3]. ETSI and ISO have suggested a globally unified mechanism for enhancing the V2V routing. Multiple accessing techniques can support the Intelligent Transportation System collaboratively [4]. But vertical handover becomes a major problem needed to be well solved in this suggestion. Research in [2] and [4] mentioned using D2D in vehicular networks. Nevertheless, in these works D2D technology has not been well coupled with the cellular network. The advantages of D2D cannot be fully used by vehicular networks.

As one of the key technologies in the field of $5 \mathrm{G}$ communication, D2D has been widely studied. Routing is the key issue in D2D multi-hop communication, which has become one of the hot topics in the context of D2D communications. Recently, Shortest Path Routing (SPR) [5], Interference Aware Routing (IAR) [6] and optimum path based routing [7] methods have been suggested. IAR aims at reducing the interference between D2D and base stations. However, due to the complexity of interference coordination algorithms and the non-standardized transmission range of signals, IAR is affected heavily by the environments. The optimum path based routing tries to find an optimum D2D path where the interference from the cellular links is within a certain value. In [8], a cluster-based multicast mode has been proposed. The cluster heads forward the multimedia data to the cluster members by using the method of multicasting. In short, the current work on D2D routing aims at simple scenarios with static nodes, such as concert halls, promotion areas in the shopping centres, etc. The high speed mobility of the nodes has not been considered yet.

In this paper, we propose a cluster-based D2D routing method aiming at solving the problem of multi-hop communications of D2D in more complex scenarios, such as 
vehicular networks where the nodes move in high speeds. During the clustering, the network assisted D2D communication is applied. The base stations provide the necessary control information to the D2D nodes, such as time synchronization, etc. Whereas not much data traffic load will brought to the base stations. In this way, large numbers of mobile nodes with high speed in a large area can be connected in the way of D2D. This can increase not only the connectivity and scalability of the vehicular networks, but also the throughput of the whole $5 \mathrm{G}$ network. The rest of the paper is organized as follows. In section II, we analyze the requirements on D2D routing from the vehicular networks. Then, we describe the cluster-based routing method in section III. We evaluate the performance of the suggested method in section IV and make conclusions in section V.

\section{REQUIREMENTS ON D2D ROUTING FROM VEHICULAR NETWORKS}

Compared with the Ad Hoc networks, vehicular networks have the following characteristics:

- Large scale. With the development of automobiles, large numbers of vehicles are on the road transporting goods and people to the destinations. Correspondingly, there is a huge number of nodes in the vehicular network. How to transmit data among the moving nodes with acceptable performance and user experience is a challenge for a vehicular network.

- Highly dynamicity. The typical speed of various vehicles on road may vary from $30 \mathrm{~km} / \mathrm{h}$ to $200 \mathrm{~km} / \mathrm{h}$, and the networking capabilities may be different. As a result, the topology of the constructed network changes very fast. A vehicular node may have connections with other nodes for only a short time. The connections in the vehicular network are intermittent.

- Limited mobility pattern. Since the running of the vehicles are limited by roads, intersections and obstacles, the mobility model of the nodes in the corresponding vehicular networks needs to be correlated with various road maps in different places, which is not easy in practice.

- Intermittent connectivity. Due to the restriction of the number of vehicles in certain areas, and the speed of the vehicles, there is not always direct connectivity between two vehicles. Sometimes a vehicle may be isolated that it does not have any vehicle neighbours in the direct communication range, and has a connection only to the base stations.

Considering the above mentioned characteristics of vehicular networks, the D2D routing mechanisms should be scalable, easy to deal with the increasing number of the network nodes. At the same time, the mobility of the individual nodes should be considered together with their locations on the road. In addition, the $\mathrm{D} 2 \mathrm{D}$ routing mechanisms should use the communication among individual vehicles as much as possible, and keep the data load for $5 \mathrm{G}$ base stations as less as possible.

To fulfil these requirements, we propose a clustering-based D2D routing method for supporting vehicular networks. Because clustering can simplify the network topology by partitioning and layering the large scale networks with different speed and connecting capabilities. This helps to setup a large scale network with acceptable networking performance. In addition, clustering may help to organizing vehicular nodes with similar speed, connectivity and running directions, which can deal with the mobility model problem, and will also benefit to establish a relatively stable network topology and connectivity.

In addition, after dividing the vehicles into clusters, the base stations can only be responsible for the communication with cluster heads or isolated vehicles, and the radio resource management and the topology management of the whole network, which can reduce greatly the data load for $5 \mathrm{G}$ base stations.

\section{A D2D Routing Method BAsed on Cluster}

The proposed D2D routing protocol consists of two procedures - clustering and routing. The clustering procedure divides the mobile nodes into groups, each with a cluster head. The network assisted D2D communication is applied during clustering. The routing procedure is responsible for forwarding packets among and within the clusters. According to the position of the nodes, the base station may or may not be involved in the routing during this procedure.

\section{A. Clustering based on Communication Range and Mobility (CCRM)}

In order to describe the mobility of vehicles, including their speed and directions, we define the following coordinate system $C$. The origin is the $(0,0)$ in the GPS coordinate, the direction of the $\mathrm{x}$-axis is from west to east, and that of the $\mathrm{y}$ axis from south to north. Therefore, each vehicle on the road has a position denoted by $\mathbf{P}(x, y), x, y$ are the latitude and longitude in the GPS coordinate, respectively; and velocity is denoted by $\mathbf{V}\left(v_{x}, v_{y}\right), v_{x}, v_{y}$ are the components of the speed along the $\mathrm{x}$-axis and $\mathrm{y}$-axis, respectively.

The clustering is divided into three steps, 1) D2D discovery; 2) grouping; and 3) cluster head selection and cluster establishment.

\section{1) $\mathrm{D} 2 \mathrm{D}$ discovery}

After a vehicle node has registered and authenticated by the mobile network through a base station, it will get a random identifier (ID). It can also obtain its velocity $\mathbf{V}\left(v_{x}, v_{y}\right)$ and position $\mathbf{P}(x, y)$ through for example the GPS system. When a vehicular node receives the D2D discovery staring signal (see section III.C), it will exchange the identity information with the nearby neighbours in a certain time slot, including ID, $\mathbf{V}$ and $\mathbf{P}$, its connectivity (see below), communication radius, and states (member or head of a cluster, etc.).

After a node $i$ with the position $\mathbf{I}\left(x_{i}, y_{i}\right)$ and velocity $\mathrm{V}_{\mathbf{i}}\left(v_{x i}\right.$, $v_{y i}$ ) gets the identity information from a neighbour node $j$ with the position $\mathbf{J}\left(x_{j}, \mathrm{y}_{\mathrm{j}}\right)$, and velocity $\mathbf{V}_{\mathbf{j}}\left(v_{x j}, v_{y j}\right)$, the node $i$ will calculate the following parameters:

\section{Relative distance $D_{i j}$ :}

The relative position $\left(X_{i j}, Y_{i j}\right)$ between node $i$ and node $j$ is: 


$$
\left(X_{i j}, Y_{i j}\right)=\left(x_{j}-x_{i}, y_{j}-y_{i}\right)
$$

Therefore, the relative distance $D_{i j}$ between node $i$ and node $j$ is:

$$
D_{i j}=\left|\left(X_{i j}, Y_{i j}\right)\right|=\sqrt{\left(x_{j}-x_{i}\right)^{2}+\left(y_{j}-y_{i}\right)^{2}} \quad .
$$

Relative velocity $\mathbf{V}_{\mathrm{ij}}$ of node $i$ and node $j$ :

$$
\mathbf{V}_{\mathrm{ij}}=\left(V_{i j x}, V_{i j y}\right)=\left(v_{x j}-v_{x i}, v_{y j}-v_{y i}\right) \text {. }
$$

The angle $\theta_{i j}$ between $V_{i}$ and $V_{j}$ :

$$
\theta_{i j}=\arccos \frac{v_{x i} v_{x j}+v_{y i} v_{y j}}{\sqrt{v_{x i}^{2}+v_{y i}^{2}} \cdot \sqrt{v_{x j}^{2}+v_{y j}^{2}}} .
$$

Suppose $R$ is the minimum communication radius of node $i$ and $j$. In order to get the time of connectivity keeping between node $i$ and $j$, we calculate the coordinates of intersection point $C(x, y)$ when node $j$ moves out of the communication range of $i$, namely:

$$
\begin{aligned}
& x=\frac{\alpha\left(\alpha X_{i j}-Y_{i j}\right) \pm \sqrt{R^{2} \cdot\left(1+\alpha^{2}\right)-\left(\alpha X_{i j}-Y_{i j}\right)^{2}}}{1+\alpha^{2}} \\
& y=\alpha x-\alpha X_{i j}+X_{i j}
\end{aligned}
$$

Here, $\alpha$ is the slope of the moving trajectory of node $j$, i.e., $\alpha=v_{y j} / v_{x j}$. In addition, as shown in Fig. 1, if $v_{x j}>0$, then the "addition" operation is used for $\mathrm{x}$. if $v_{x j}<0$, the "subtraction" is used to $x$. If $v_{x j}=0$, the moving path of $j$ is perpendicular to the $\mathrm{x}$-axis. In this case, the result of $C(\mathrm{x}, \mathrm{y})$ is simplified to (5).

$$
\begin{aligned}
& x=X_{i j} \\
& y= \pm \sqrt{R^{2}-X_{i j}^{2}}
\end{aligned}
$$

\section{The connection keeping time $\boldsymbol{T}_{i j}$ between node $i$ and $j$ :}

Since node $j$ will move out of the communication range of node $i$ in the relative speed of the two nodes at the intersection point $C$, the time $T_{i j}$ that the connection between node $i$ and $j$ can be kept is:

$$
T_{i j}=\frac{\sqrt{\left(x-X_{i j}\right)^{2}+\left(y-Y_{i j}\right)^{2}}}{\sqrt{v_{i j x}^{2}+v_{i j y}^{2}}}
$$

\section{2) Grouping}

The principle for establishing clusters is to keep all the clusters as stable as possible. In other words, a cluster head and its member nodes should be able to communicate with each other as long as possible when all the vehicles are moving at different velocities. Therefore, before a cluster is formed and the head is selected, the nearby nodes are first grouped according to their relative position and relative velocity. To keep a stable and reliable connection between the nodes, the vehicular node $i$ and $j$ will be classified into one group if the following three conditions are satisfied:

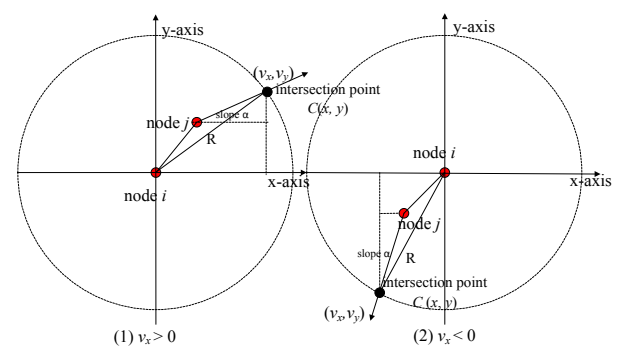

Fig. 1. Relative moving velocity and connection time between node $i$ and $j$. a) The distance between two nodes is less than $50 \%$ of the communication range $\mathrm{R}$;

b) The angle between the moving direction of the two nodes, i.e., the velocity angle $\theta$ is less than the threshold angle $\theta_{t h}$. ( $\theta_{t h}$ is set to $45^{\circ}$, see section IV);

c) The connection time between two nodes is larger than the threshold value $T_{t h}$.

The purpose that the node distance s less than $50 \%$ of the communication range is to guarantee that nodes within one cluster can communicate with each other directly, and also the neighbouring cluster heads can communicate with each other directly. $T_{\text {th }}$ can be determined according to the stability requirement of the clusters or the average connectivity time among the vehicles in the network. In our simulations, $T_{t h}$ $=0.5 *\left(T_{\text {th-old }}+T_{\text {average }}\right)$. Namely, we calculate $T_{\text {th }}$ periodically according to the past and current average connectivity time among the vehicles.

\section{3) Cluster head selection and cluster establishment}

The main procedure for establishing a cluster is to select a cluster head in each group, and announce it to all other nodes in the group. In order to make a cluster stable and have efficient communications, a cluster head should have as many direct connections with its members as possible and can maintain the communication possibility as long as possible. Hence, we introduce the conception of connectivity of a node, which is defined as the number of neighbour nodes satisfying the above three grouping conditions.

Namely, given a node $c$ and any node $n$, according to the results of formula (1) to (5), during the grouping period the distance $D_{c n}$, the moving angle $\theta_{c n}$, and the connectivity keeping time $T_{c n}$ between the two nodes can be calculated. If $D_{c n}, \theta_{c n}$ and $T_{c n}$ satisfy the grouping conditions, the value of the connectivity $d_{c}$ of node $c$ will increase by one. Thus, when the grouping phase is finished, each node can get its connectivity value $d_{c}$. The procedure for establishing clusters is shown in Fig. 2.

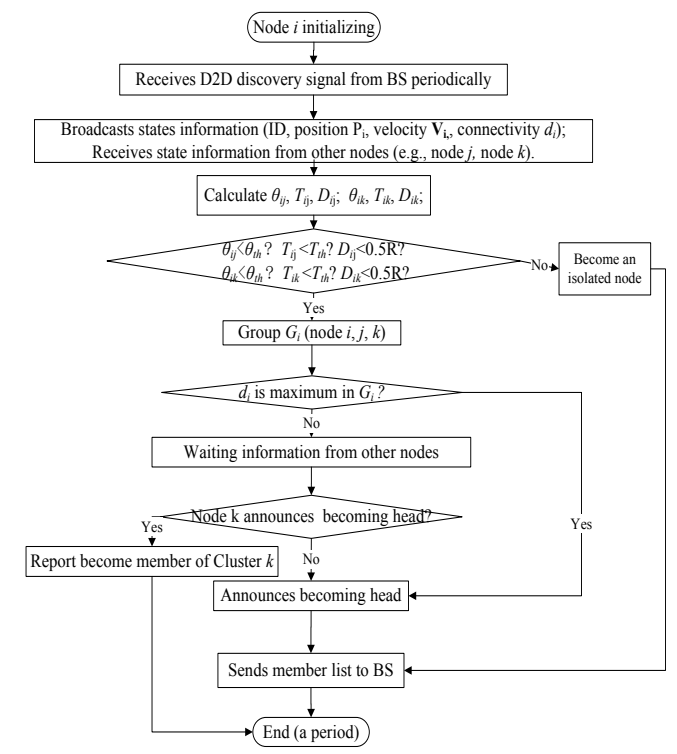

Fig. 2. Procedure for clustering. 
After clusters are formed, the nodes need to exchange their membership and states information periodically. Cluster heads exchange its member list with base stations periodically, and also broadcast the member list to the neighbour cluster heads.

If multiple base stations are involved in a vehicle network, the cluster information needs to be synchronized among base stations. The base stations exchange with each other the information about the clusters within their own coverage periodically. In this way, each base station has the information about the whole vehicular network.

\section{4) Cluster mainteinence}

Due to the mobility of the vehicles, the clusters are changing dynamically - the existing members or even the head may leave, and new members may join the cluster; a cluster may split into several clusters; two clusters may also merge into one cluster.

Every cluster head exchanges state information with its members periodically. If a cluster head has not received any information from a member for a certain period, the cluster head will update its member list, broadcast it to its members and synchronize it with the base stations. For a node that has not got any information from a cluster head for a certain time, due to its or the cluster head's leave, it will start the procedure of joining another cluster or become a cluster head during the D2D discovery and grouping period, as shown in Fig. 2.

If a member node $i$ with current cluster head $j$ receives broadcasting message from another cluster head $k$, the node $i$ will calculate the connection keeping time $T_{i j}, T_{i k}$ and the velocity angle $\theta_{i j}$ and $\theta_{i k}$. If $T_{i j} \geq \beta T_{i k}(\beta \geq 1)$, and $\theta_{i j} \leq \gamma \theta_{t h}(\gamma \geq 1)$, the node $i$ will stay in the old cluster. Otherwise, if the above conditions cannot be satisfied for several periods, the node will swap to cluster $k$ by broadcasting its cluster ID $=k$. The cluster head $k$ will accordingly update its member list in a certain time period.

During the Inter-cluster communication period (see section $\mathrm{C}$ below), the cluster head $j$ broadcasts its states and member list, and receives information from neighbour clusters. If the distance between two cluster heads are shorter than the clustering radius $R$, the cluster head $j$ who has fewer members than cluster head $k$ will initiate the cluster merging procedure. Then, during the Intra-cluster communication period, the cluster head $j$ will notify its members to move to cluster $k$ by changing the cluster ID to $k$.

If a cluster head does not want to act as head or the number of its members becomes more than a threshold, it will broadcast a message to all its members or part of its member of dismissing a cluster. The members who got the message will start the procedure of joining another cluster or become a cluster head during the D2D discovery and grouping period.

\section{B. Routing based on Clusters}

According to the position and the roles of nodes after clustering, the routing can be classified into three categories: routing within a cluster; routing within the inter-connected clusters; and routing among isolated clusters.

After clusters are formed, a cluster head broadcasts the member list to all its members and each member has a member list. Therefore, if a node finds its communication partner is within the same cluster, it will initiate a communication request directly to its partner. Since within a cluster, the distance between two nodes are less than $50 \%$ of the maximum wireless transmission range, therefore, within a cluster, the packets can arrive at the destination by one hop.

If the source and the destination are in different clusters, one or more base stations may be involved in the communications. After the clusters are formed, each cluster head can obtain the member list of the neighbour clusters, and be broadcasted to its neighbours. As a result, each node has a neighbour table maintaining the nodes in its own cluster and the neighbour clusters within one hop. And the base station has the information of all the nodes after the synchronization among the base stations. Fig. 3 shows the communication between two nodes in the different clusters inter-connected with each other. Since the base station has all the nodes' information, it can decide if one or more base stations are needed to be involved in the routing according to the number of the hops required.

If two clusters are isolated, the base station cannot find a path for two nodes belonging to each of them without involving the base stations themselves. Therefore, after recalculating the path possibly involving several base stations, the base station will send the path to the corresponding nodes.

\section{D2D Media Access Control based on Clusters}

The media access control procedure of the above D2D communication, including the control and data transmission, is illustrated in Fig. 4. Similar to work [8], in each communication period, during the Synchronization phase (Sync. Phase), the registration of UEs, time synchronization between UEs and the base station, etc. are performed. During the device discovery and identification phase (Discovery

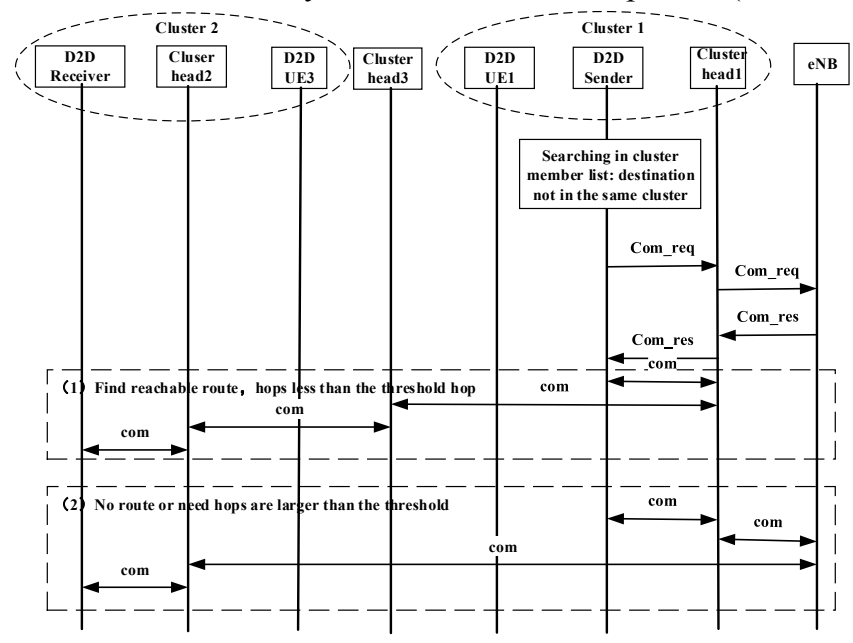

Fig. 3. Multiple hops communication between nodes in inter-connected clusters. 
Phase), first, the base station sends D2D discovery signal to all the UEs within its coverage. Then, the UEs send discovery signal to each other, obtaining the states of each other, and calculating the parameters related to clustering. During the control phase (Control Phase), the clustering will be finished and the wireless resources are allocated. As shown in Fig. 4, the control phase is further divided into five sub-phases. During the Cluster Formation Phase, the cluster heads are selected and the clusters are formed. The wireless resources will be allocated to the cluster heads and their members during the eNB Control Phase 1. During the Inter-Clusters Control Phase, the information among the clusters is exchanged. During the Intra-Cluster Phase, the management information exchange within a cluster is performed. The necessary information among nodes, such as routing information, is exchanged during the eNB Control Phase 2. The communication among nodes is executed during the Communication Phase.

\section{Performance Evaluation}

We implemented the proposed method in the network simulator ns-3 [9]. To evaluate the performance of the proposed method, we used the tool OpenStreetMap (OSM) [10] and Simulation of Urban Mobility (SUMO) [11] to create different mobility scenarios. OSM provides freely exportable maps of cities, which is commonly regarded as the highest quality road data publicly available today. SUMO is an opensource traffic simulator with continuous space and discrete time and is capable of importing maps in multiple formats, including OSM. In our simulation, we used OSM to create the map of the real-world, and SUMO to generate vehicle nodes, traffic and mobility model in the maps with real-world roads. The results are used as input of ns-3. Fig. 5 shows one of the maps we used in our simulation. Fig. 6 illustrates the clustering results at a certain time point when there are 200 vehicular nodes on the roads.

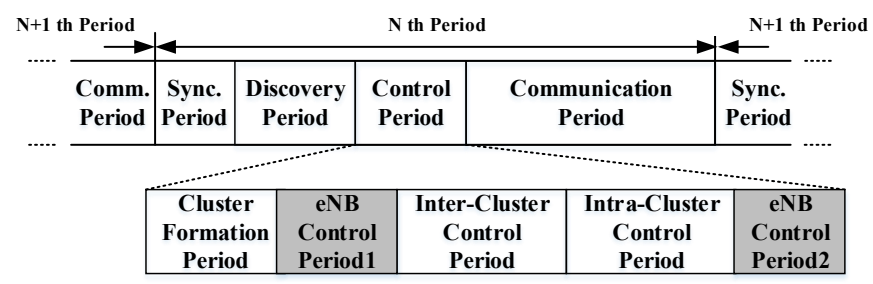

Fig. 4. Control period for clustering-based D2D routing.

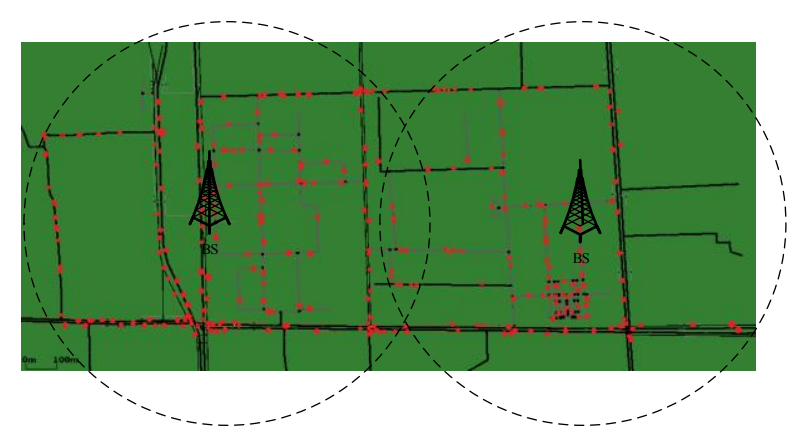

Fig. 5. Map and distribution of vehicles at a certain time point (200 vehicular nodes).

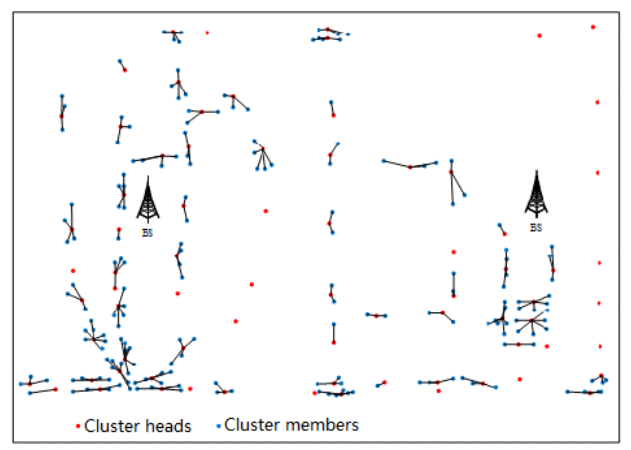

Fig. 6. Results of clustering at a certain time (200 vehicular nodes).

To evaluate the stability of clusters by using our CCRM algorithm, we tested the cluster changes and the numbers of existing clusters with the running of time under different velocity angles, as illustrated in Fig. 7 and Fig. 8. Note that whenever a member leaves or joins a cluster, or a head leaves or emerges, we increase the number of cluster change by one. Here, we can see that when the relative velocity angle is restricted to $10^{\circ}$, the number of cluster changes increases much slowly than when the angle is restricted to $90^{\circ}$, and when the angle is $45^{\circ}$, the number of cluster changes does not increase much. However, if the velocity angle is restricted to $10^{\circ}$, the total number of clusters is the most. This may increase the burden of the base stations. Thus, we set the velocity angle of our algorithm to $45^{\circ}$ in the following tests.

Fig. 9 illustrates the average time for nodes to keep as cluster heads. Here, we can see that by using our CCRM algorithm, the cluster heads did not change as frequently as the Minimum ID algorithm [12] and the highest-degree connectivity-based clustering algorithm [13], which are the two typical clustering methods for Ad Hoc networks.

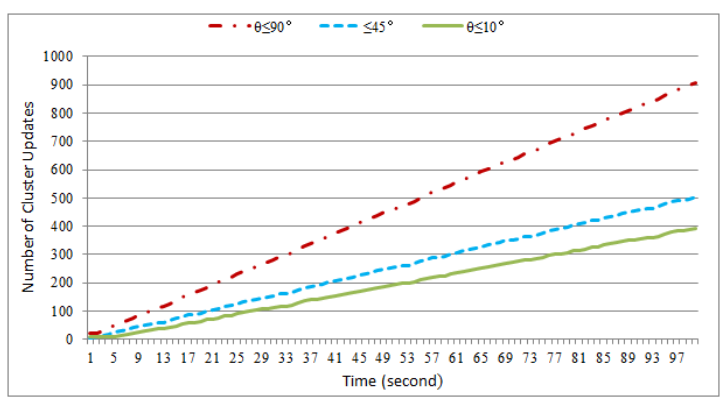

Fig. 7. Changes of clusters under different velocity angle restrictions.

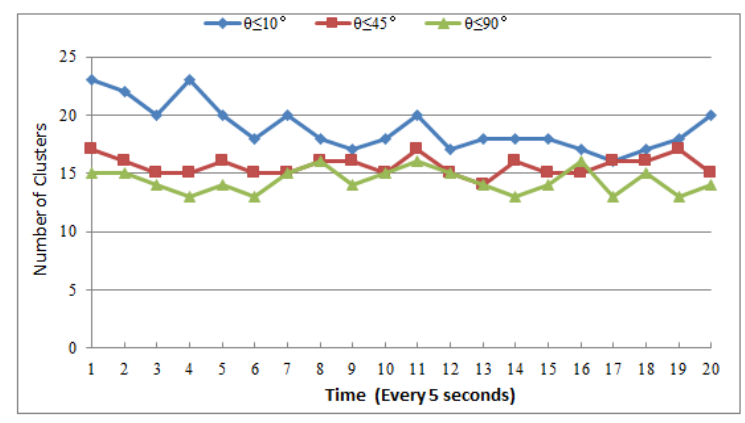

Fig. 8. Number of clusters under different velocity angle restrictions. 


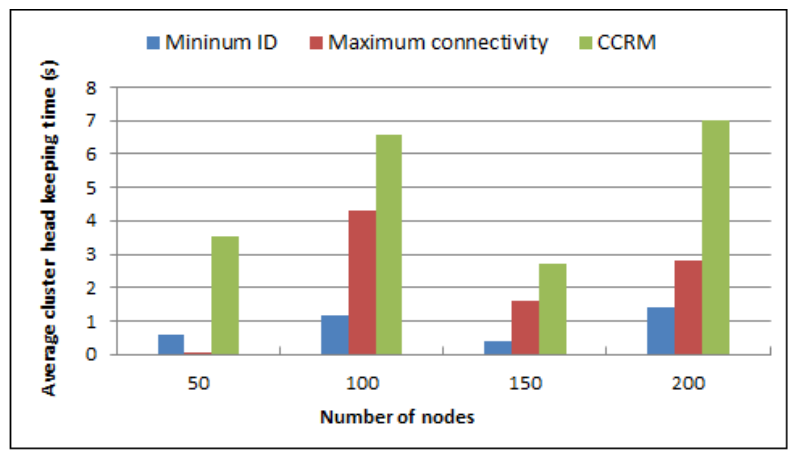

Fig. 9. Average time of nodes keeping as cluster heads.

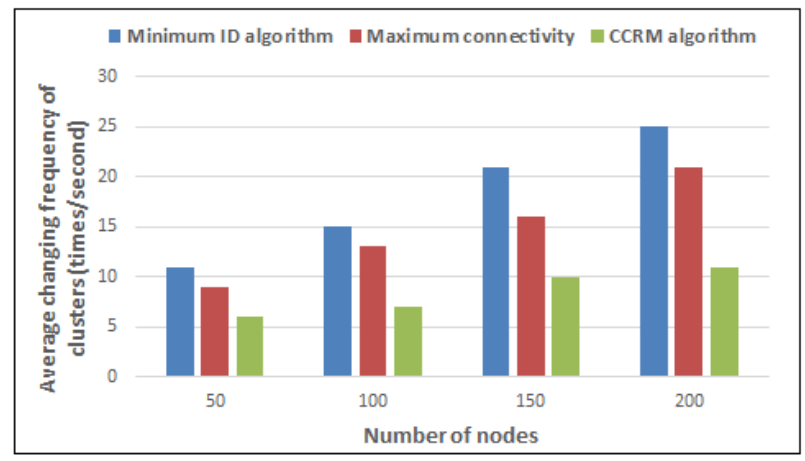

Fig. 10. Frequncy of cluster changes.

Fig. 10 shows the average frequencies of cluster changes, including the joining and leaving of both the cluster members and heads, compared with the Minimum ID algorithm and the Maximum Connectivity algorithm. Here, we can see that by using our CCRM algorithm, the clusters are more stable than those obtainable by using the other two algorithms.

Figure 11 illustrates the overhead of the routing method based on CCRM. A source node sends packets every 0.3 seconds for 100 seconds. We measured the number of the routing control related message $n_{r}$ and the number of the message for packet transmission $n_{d}$ in the network. Here, the overhead is defined as the ratio of $n_{r}$ to $n_{r}+n_{d}$. We compared the routing based on CCRM with CGSR (Clusterhead Gateway Switch Routing) [14] and ZRP [15] (Zone Routing Protocol), which are typical hierarchical routing protocols.

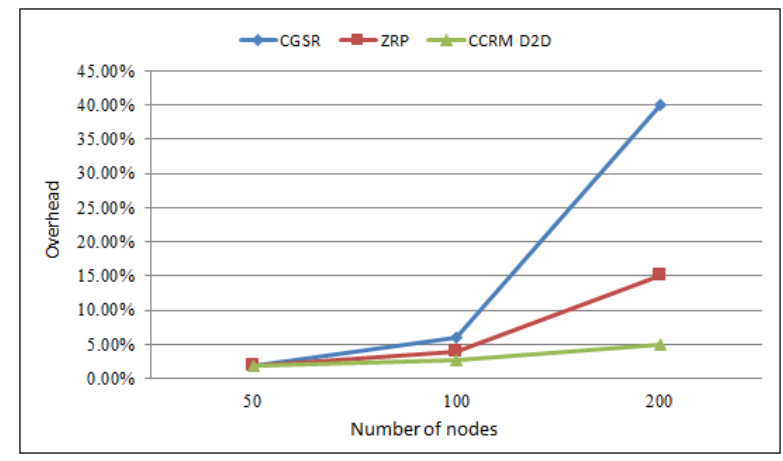

Fig. 11. Overhead of different routing methods

\section{CONCLUSIONS}

In this paper, we propose a cluster-based D2D routing method oriented to vehicular networks. Vehicular nodes are clustered according to their relative running speed and direction. The base stations provide the necessary control information to the D2D nodes, such as time synchronization, etc., and not much data traffic load will brought to the base stations. In this way, large numbers of mobile nodes with high speed in a large area can be connected in the way of D2D. This method can increase not only the connectivity and scalability of the vehicular networks, but also the throughput of the whole $5 \mathrm{G}$ network. We have analysed our method by using the real world map and dynamic generated traffic nodes. The results show that our method is stable and inexpensive, and is suitable for the vehicular networks. Our future work is to make a more thorough analysis and evaluation of the proposed algorithm.

\section{REFERENCES}

[1] E. Abdelrahman, A. Said, T. Toukabri, et al. "A Hybrid Model to Extend Vehicular Intercommunication V2V through D2D Architecture", in proc. of International Conference on Computing, Networking and Communications (ICNC). 2015(12):754-759.

[2] W. Xing, N. Wang, C. Wang, et al. "Resource Allocation Schemes for D2D Communication used in VANETs", in proc. of Vehicular Technology Conference (VTC 2014 Fall). 2014(9): 1-6.

[3] A. Khelil, D. Soldani, "On the Suitability of Device-to-Device Communications for Road Traffic Safety", in proc. of IEEE World Forum on Internet of Things, 2014, 16(1): 224-229.

[4] K. Vanganuru, S. Ferrante and G. Sternberg, "System Capacity and Coverage of a Cellular Network with D2D Mobile Relays", in proc. of Military Communications Conference, 2012, pp. 1-6.

[5] S. Wang, W. Guo and Z. Zhou, et.al, "Outage Probability for Multi-Hop D2D Communications With Shortest Path Routing", IEEE Communications Letters. 2015(9), 19(11):1997-2000.

[6] G. Parissidis, M. Karaliopoulos, T. Spyropoulos, et al, "InterferenceAware Routing in Wireless Multihop Networks", IEEE Transactions on Mobile Computing. 2010(10): 716-733.

[7] V. Bhardwaj and C.R. Murthy, "On Optimal Routing and Power Allocation for D2D Communications", in proc. of IEEE International Conference on Acoustics, Speech and Signal Processing (ICASSP), 2015(4): 3063 - 3067.

[8] J. S. Kim, J. Gu, Y. C. Min, et al, “ A Novel Medium Access Scheme for Cluster Based Device-to-Device Broadcast Communications", in proc. of International Conference on Information Networking. 2015(11): 397-401.

[9] ns-3: A Discrete-event Network Simulator. http://www.nsnam.org/

[10] OpenStreetMap._http://www.openstreetmap.org

[11] Simulation of Urban Mobility. http://sumo.sourceforge.net

[12] M Gerla, TC Tsai. Multicluster, Moblle, Multimedia Radio Network[J]. Wireless Networks, 2010, 1(3): 255-265.

[13] M Chatterjee, SK Das, D Turgut. WCA:A Weighted Clustering Algorithm for Mobile Ad hoc Networks. Clustering Computing. 2002,5(2): 193-204.

[14] C.-C. Chiang, "Routing in Clustered Multihop, Mobile Wireless Networks with Fading Channel" Proc. IEEE SICON'97, Apr.1997, pp.197-211.

[15] Z. J. Haas, M. R. Pearlman and P. Samar, "The Zone Routing Protocol for Ad Hoc Networks (ZRP)", IETF draft, July 2002.

Fig. 11. Overhead of different routing methods 